\title{
M\&MoCS - International Research Center on Mathematics and Mechanics of Complex Systems
}

Francesco dell'Isola (University of Rome "La Sapienza", Italy and International Research Center on Mathematics and Mechanics of Complex Systems, L'Aquila, Italy), Luca Placidi (International Telematic University Uninettuno, Italy and International Research Center on Mathematics and Mechanics of Complex Systems, L'Aquila, Italy) and Emilio Barchiesi (University of Rome "La Sapienza", Italy and International Research Center on Mathematics and Mechanics of Complex Systems, L'Aquila, Italy)

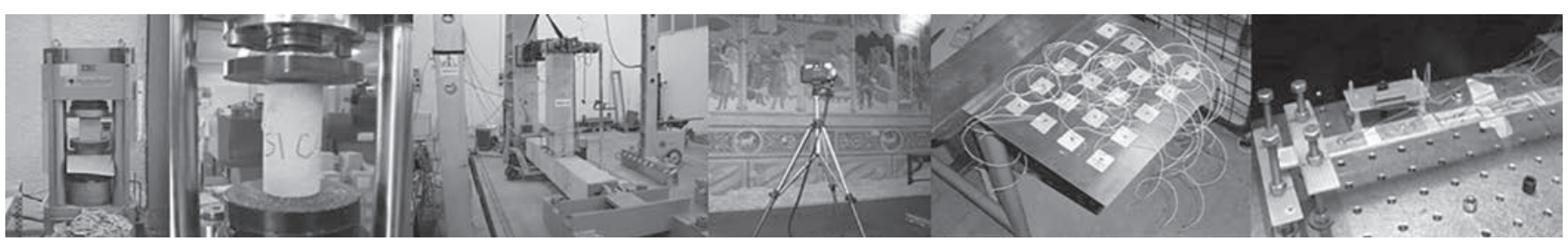

\section{The Centre}

The International Research Center for Mathematics \& Mechanics of Complex Systems (M\&MoCS) is a research centre of the University of L'Aquila. The centre was established in 2010 by the Dipartimento di Ingegneria delle Strutture, delle Acque e del Terreno (DISAT), the Dipartimento di Matematica Pura e Applicata (DMPA) of the University of L'Aquila and the Dipartimento di Strutture of Roma Tre University, with the financial and logistic aid of Fondazione Tullio Levi-Civita. The current director of the centre is Francesco dell'Isola, who is a full professor of mechanics of solids at "La Sapienza" University of Rome. The previous director of the centre was Angelo Luongo, who is a full professor of mechanics of solids at the University of L'Aquila.

The mission of the centre is the development and dissemination of scientific knowledge. In order to pursue these goals, the centre: (a) conducts and coordinates research activities; (b) promotes initiatives for the enhancement of scientific liaison between researchers in mathematical fields and researchers in solid and fluid mechanics, operating both in Italy and abroad; (c) promotes, supports and organises highly qualified educational activities, such as training, Master's and doctorate courses; (d) encourages the promotion of mathematics and mechanics of complex systems through publications, conferences, seminars and exhibitions; and (e) carries out consultancy and research activities for organisations and institutions.

\section{Workshops and summer schools}

From 2011 to 2013, the centre offered the Sperlonga Summer Schools on Mechanics and Engineering Sciences: courses and seminars, organised with an interdisciplinary attitude, aimed at introducing young scientists to present-day developments in mechanics at the interface with mathematics, physics, materials science, biology and engineering. Lectures were complemented by discussion sessions to foster lively interactions amongst participants. Moreover, the centre, in collaboration with the CNRS International Associate Laboratory Coss\&Vita, the Paris Federation of Mechanics Labs and the GDR MeGe (French Research Network), has organised, since its foundation, many workshops in Arpino, Alghero and Catania. Since 2014, the centre has organised two EUROMECH colloquia, and a third one "Generalized and microstructured continua: new ideas in modeling and/or applications to structures with (nearly-)inextensible fibers" is planned for 3-8 April 2017 in Arpino (Italy). In the framework of a joint effort with Warsaw University of Technology and among the many seminars organised at the centre, an introductory course in analytical continuum mechanics and computational mechanics is offered every year. Many other events are scheduled throughout the year.

To find out more information, please visit the website http://memocs.univaq.it.

\section{Research and facilities}

Research activities carried out at the centre are directed toward the formulation of computationally tractable mathematical models to predict phenomena occurring in complex systems and address their numerical solution. Experimental research is also being carried out at the centre facilities. The research mainly concerns: variational and optimisation methods, gamma convergence, homogenisation techniques for periodic media, mechanics of fluids and solids, vibration control by means of piezoelectric actuators, composite materials, landmine detection, biomechanics of growing tissues, fluid dynamics and transport phenomena, kinetic theory, vibrations and waves in continuous and multi-phase media, plasticity, damage mechanics, continuum mechanics, stability and control of structures, identification of materials and 
mechanical systems, dynamical systems and bifurcation theory, fluid dynamics models for the analysis of traffic flows and the social sciences, and numerical differential modelling of the mechanical and electromagnetic response of biological materials and nano-structures.

In the following subsections, we briefly summarise the main activities of the experimental laboratories.

\section{Laboratory of Materials and Structures Testing}

The activities of the Laboratory of Materials and Structures Testing concern experiments on materials and structures for the purpose of consultancy, applied research and teaching. The aim of the laboratory is to provide the construction industry with a diagnosis of the state of degradation of civil works, thus providing an assessment of the residual life of structures.

\section{Laboratory of Structures and Smart Materials}

The Laboratory of Structures and Smart Materials is actively engaged in the study and prototyping of smart structures. The research group addresses the issue of mechanical structure vibration damping using piezoelectric transducers coupled with electronic systems and the research is directed toward linear and nonlinear control of structural dynamics. Applications considered include the design of soundproofing systems, wing and blade flutter control, the identification of structural damage and the design of smart systems able to self-monitor the evolution of their constitutive parameters. Uncertainty modelling in inhomogeneous structures with unknown inhomogeneity and stimulated by piezoelectric actuators and the analysis of metals subject to the action of external loads and induced structural change (such as anisotropy and strength of material) are also investigated.

\section{Naval Structures and Onboard Instrumentation Laboratory}

The Naval Structures and Onboard Instrumentation Laboratory (officially known as Laboratorio Strutture Navali e Strumentazioni di Bordo) conducts research on prototypes of ship structures (surface and underwater vehicles). The research activity is focused on the areas of control and vibration damping in the field of marine structures and stability of ship structures subject to the action of fluid waves and impact. The laboratory is directly involved in the development and realisation of the project SEALAB. The SEALAB project includes the construction of an experimental surface marine mobile station, functioning as a test bed for marine technologies. It is intended to develop, validate, refine and eventually patent new design solutions, devices and innovative systems in the field of marine engineering. At the same time, SEALAB aims to bring together, in a single project, the efforts and skills of university and industry research. Although SEALAB was born with the goal of providing an experimental platform to develop new solutions, the vehicle being developed can be used in an HSU (high-speed unmanned) version as a coastal patrol in autonomous and/or high speed (close to $200 \mathrm{~km} / \mathrm{h}$ ) remote driving. It could potentially be employed in civil defence or for the coast guard, etc.

\section{Vibrations Laboratory}

The Vibrations Laboratory conducts research in the fields of smart structures, vibration control, noise generation and transmission, and structural integrity monitoring. In particular, research focuses on: (a) non-destructive fresco integrity diagnosis with Doppler vibrometer scanning lasers; (b) development of acoustic-vibrators for the location of historically significant fresco and plaster defects; (c) development of lighter and cheaper flexible robotic systems; (d) development of smart structures, equipped with piezoelectric sensors such as thin plates or panels for noise and vibration control; (e) finite element modelling for the design of structures in the civil and industrial sectors, such as wood laminated orthotropic structures; and (f) energy analysis of buildings.

\section{Humanitarian Demining Laboratory (HDL)}

The main aim of the Humanitarian Demining Laboratory (HDL) is to develop new anti-personnel mine detection devices for humanitarian demining. Experimental activity is being carried out on a promising original active thermal technology based on localised heating pulses and temperature sensing. With the aim of developing a multi-sensor platform employing data fusion and collaborating robotic agents, vibrometric/ acoustic and GPR techniques are also employed. For experimental purposes, a computer-controlled cart that can move over a sand box while holding a heater and other instrumentation has been realised. In the sand box, two accurate low-metal-content mine surrogates of different materials are hidden, together with another object. An outdoor "minefield" has also recently been realised.

\section{Functional Multiscale Metamaterials and Smart Systems Lab}

The activities carried out in the Functional Multiscale Metamaterials and Smart Systems Lab range from numerical modelling to experiments on micro- and nano-structural and functional materials. The materials have many applications, from biomaterials to energy harvesting. The laboratory has equipment for electron, ionic and atomic force microscopy (including advanced technologies for spectroscopy and nanoindentation) for the structural characterisation of micro- and nano- innovative materials.

\section{MEMOCS Journal}

The International Research Center for the Mathematics and Mechanics of Complex Systems has founded the homonymous journal Mathematics and Mechanics of Complex Systems, abbreviated to MEMOCS, for the benefit of the community of researchers in mechanics and mathematics. MEMOCS is peer-reviewed, indexed in all major databases and free to both authors and readers. It publishes articles from diverse scientific fields with 
a specific emphasis on mechanics. Articles must rely on the application or development of rigorous mathematical methods. Indeed, the journal is intended to foster a multidisciplinary approach to knowledge firmly based on mathematical foundations. It will serve as a forum where scientists from different disciplines meet to share a common, rational vision of science and technology. It is intended to support and divulge research whose primary goal is to develop mathematical methods and tools for the study of complexity. The journal also fosters and publishes original research in related areas of mathematics of proven applicability, such as variational methods, numerical methods and optimisation techniques. Besides their intrinsic interest, such treatments can become heuristic and epistemological tools for further investigations and provide methods for deriving predictions from postulated theories. Papers focusing on and clarifying aspects of the history of mathematics and science are also welcome. All methodologies and points of view, if rigorously applied, are considered.

To find out more information, please visit the website http://memocs.org.

\section{$\mathrm{M} \& \mathrm{MoCS}$ \\ DICEAA}

Università degli Studi dell'Aquila

Via Giovanni Gronchi 18

67100 L'Aquila, Italy

Tel. 06.90.28.67.84 Fax 0773.1871016

Website: http://memocs.univaq.it

Email:memocs.cisterna@gmail.com

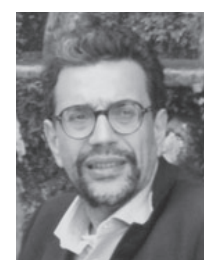

Francesco dell'Isola received cum laude his degree in physics at the University of Naples "Federico II" in 1986. At the same university, in 1992, he received a PhD in mathematical physics with a thesis on the rational thermodynamics of nonmaterial bidimensional continua. Since 2006, he has been a full professor of solid and structural mechanics at "La Sapienza" University of Rome. Professor dell'Isola has authored more than 150 papers in international journals.

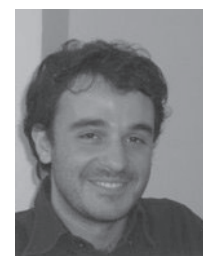

Luca Placidi graduated cum laude in physics at the University of Naples "Federico II" in 2001 and in engineering at the Virginia Polytechnic Institute in 2002. He received a PhD in 2004 from the Technical University of Darmstadt and a second one in 2006 from "La Sapienza" University of Rome. He has authored five books and more than 40 papers in journals. Since 2011, he has been an assistant professor at the International Telematic University Uninettuno.

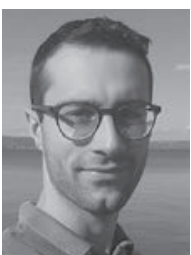

Emilio Barchiesi completed cum laude his $M S c$ in mathematical engineering at the University of L'Aquila (Italy) in 2016, defending a thesis on the numerical identification of mathematical models for the description of engineering fabrics. He is currently pursuing his PhD studies in theoretical and applied mechanics at "La Sapienza" University of Rome. His main research interests lie in homogenisation theory, computational mechanics, higher gradient continua and variational methods. 